Supplementary information for

\title{
All Solid-State Synaptic Transistors with High-Temperature Stability Using Proton Pump Gating of Strongly Correlated Materials
}

Chadol Oh, Minguk Jo, and Junwoo Son*

Department of Materials Science and Engineering (MSE), Pohang University of Science and Technology (POSTECH), Pohang 37673, Republic of Korea 

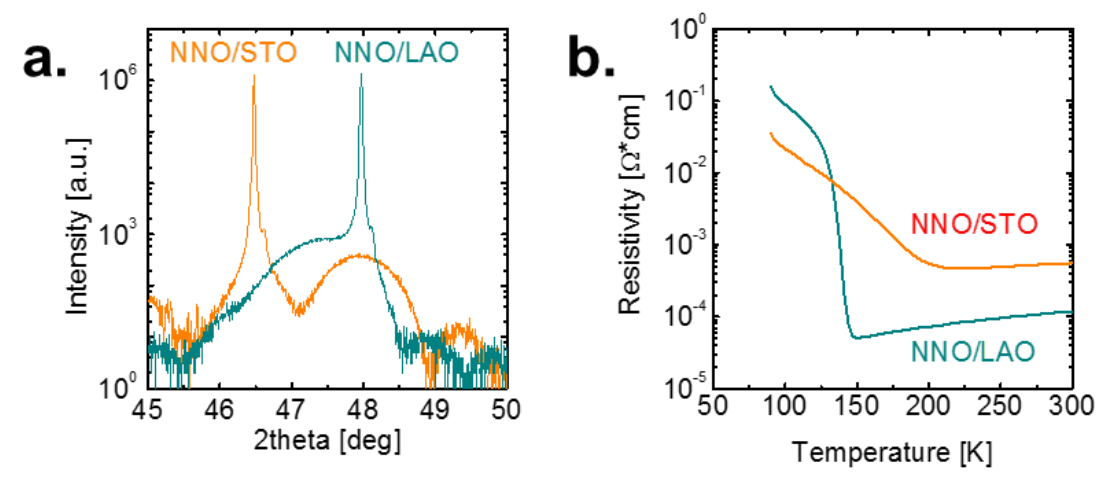

Figure $\mathrm{S} 1$ | $\mathrm{NdNiO}_{3}$ epitaxial films grown on $\mathrm{SrTiO}_{3}$ and $\mathrm{LaAlO}_{3}$ substrates for the channel

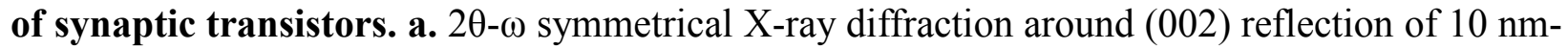
thick NNO films on (001)-oriented STO and (001) pc-oriented LAO substrates. b. Resistivity as a function of temperature of 30-nm-thick NNO films on both substrates. Temperature-dependent resistivity of both NNO films clearly showed metal-insulator transition below room temperature (transition temperature: NNO on STO: $\sim 220 \mathrm{~K}$; NNO on LAO $\sim 150 \mathrm{~K}$ ), which confirms that $\mathrm{NNO}$ as a correlated material at the phase boundary between metal and insulator due to strong correlation in $d$ orbitals 
a.

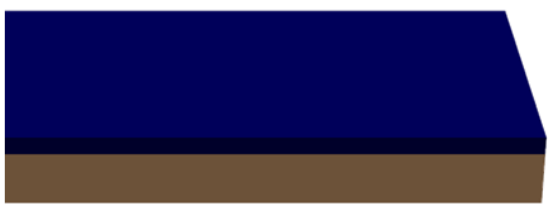

b.

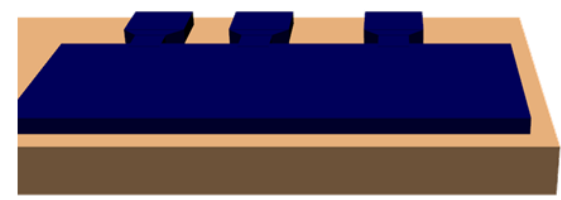

C.

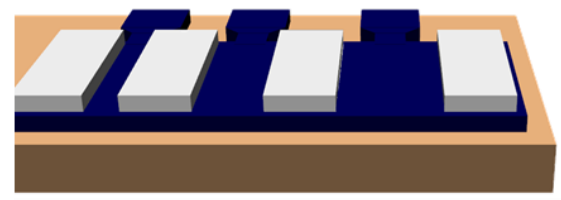

d.

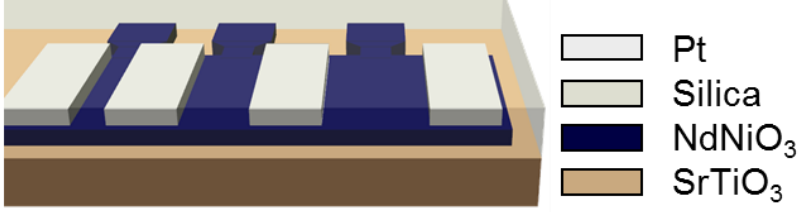

e.

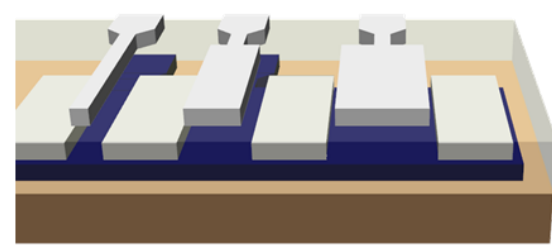

f.

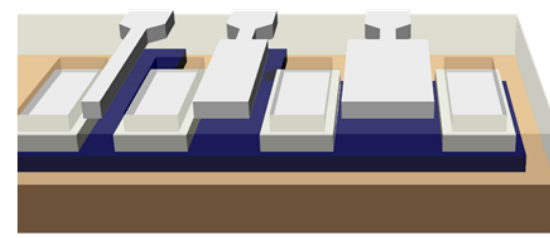

Figure S2 | Fabrication process of three-terminal synaptic transistor. a. Epitaxial growth of $\mathrm{NdNiO}_{3}(\mathrm{NNO})$ channel on substrate by using pulsed laser deposition. b. Patterning of NNO channel using positive photolithography and subsequent wet etching with $\mathrm{HCl}$ solution. c. Lift-off process for Pt source and drain electrodes. d. A 300-nm-thick porous sol-gel silica spin coating. e. Lift-off process for Pt gate electrodes. f. Wet etching of porous sol-gel silica layer to expose source and drain electrodes using 1:7 diluted buffered oxide etchant. 

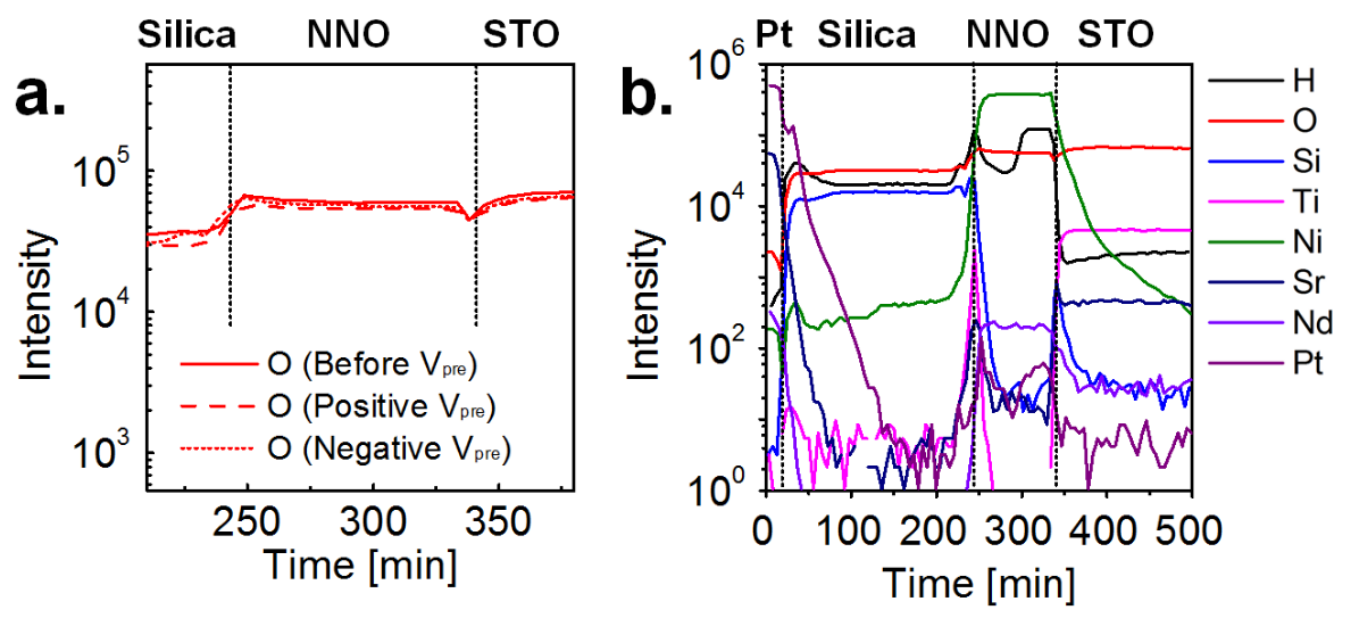

Figure S3 | Dynamic secondary ion mass spectroscopy. a. Depth profile of O ion in synaptic transistor right underneath the gate electrodes before positive $V_{\text {pre, }}$ after positive $V_{\text {pre, }}$ and after negative $V_{\text {pre. }}$ Application of $V_{\text {pre }}$ did not cause any apparent change in the signal for oxygen, so oxygen vacancies cannot be the origin of metal-to-insulator transition induced by $V_{\text {pre }}$ in our device b. Elemental depth profiles right underneath gate electrode before positive $V_{\text {pre }}$ 


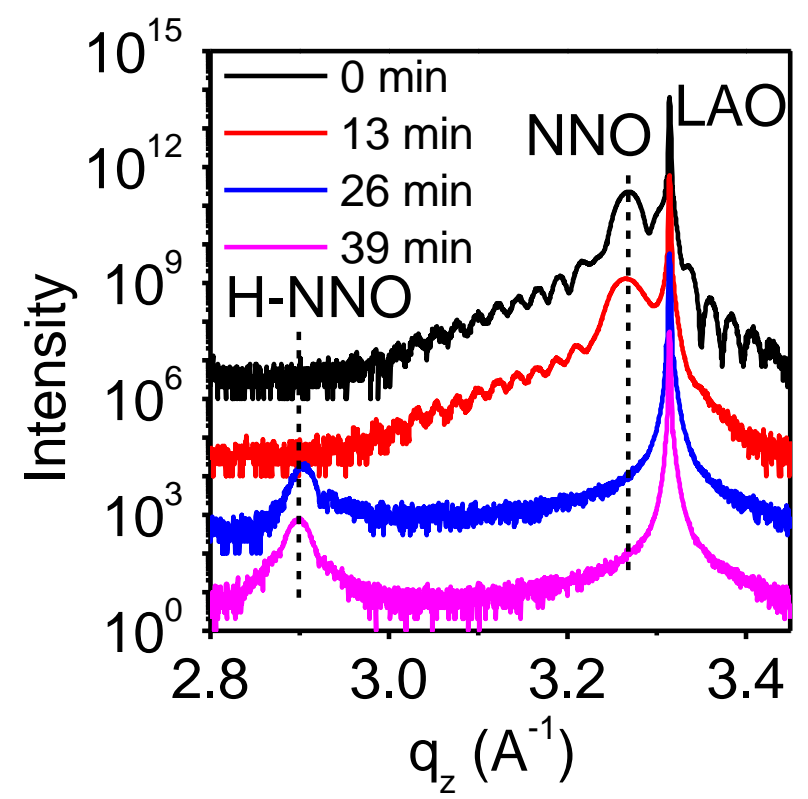

Figure S4 | Determination of X-ray diffraction peak of pristine NNO and fully-protonated NNO (H-NNO). Structural expansion during $\mathrm{H}^{+}$intercalation in NNO films was confirmed by insitu X-ray diffraction of 10-nm-thick NNO thin film with Pt nanoparticles under hydrogen spillover during exposure to $5 \% \mathrm{H}_{2} / 95 \%$ Ar forming gas. The initial peak before hydrogenation was detected at a $\mathrm{q}_{\mathrm{z}}$ value of $3.27 \AA^{-1}$, which denotes diffraction from the (002) plane in pristine NNO films. When the sample was exposed to the forming gas to incorporate hydrogen interstitials by hydrogen spillover, the peak intensity of pristine $\mathrm{NNO}$ at $\mathrm{q}_{\mathrm{z}}=3.27 \AA^{-1}$ gradually decreased with time and disappeared within 26 min; afterward, a new peak appeared at $\mathrm{q}_{\mathrm{z}}=2.89 \AA^{-1}$, which represents $\mathrm{H}^{+}$-induced lattice expansion in $\mathrm{H}-\mathrm{NNO}$ phase. The peak intensity is slightly lower and wider in $\mathrm{H}-\mathrm{NNO}$ phase than in NNO phase; this difference indicates a loss of coherent $\mathrm{x}$-ray diffraction due to inferior crystal alignment of H-NNO phase along the (001) direction after hydrogenation. 

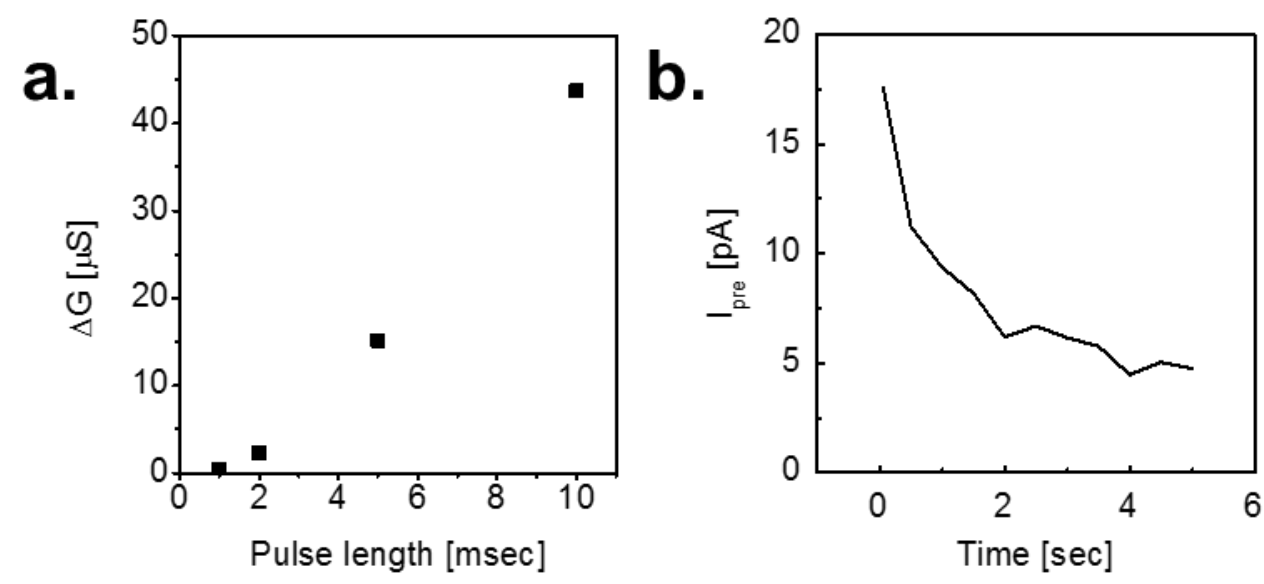

Figure S5 | Synaptic behavior in our proton transistors at $100^{\circ} \mathrm{C}$. a. Conductance change as a function of pulse length with $4 \mathrm{~V}$ of pulse amplitude. b. Time-dependent $I_{\text {pre }}$ measured by applying $50 \mathrm{mV}$. The energy consumption per synaptic event can be estimated by calculating $\mathrm{d} E=V_{\text {pre }} \times I_{\text {pre }}$ $\mathrm{x} d t$ and integrating over time. Thus, the energy consumption was obtained by integrating the area of b. then multiplying by $V_{\text {pre. }}$ 

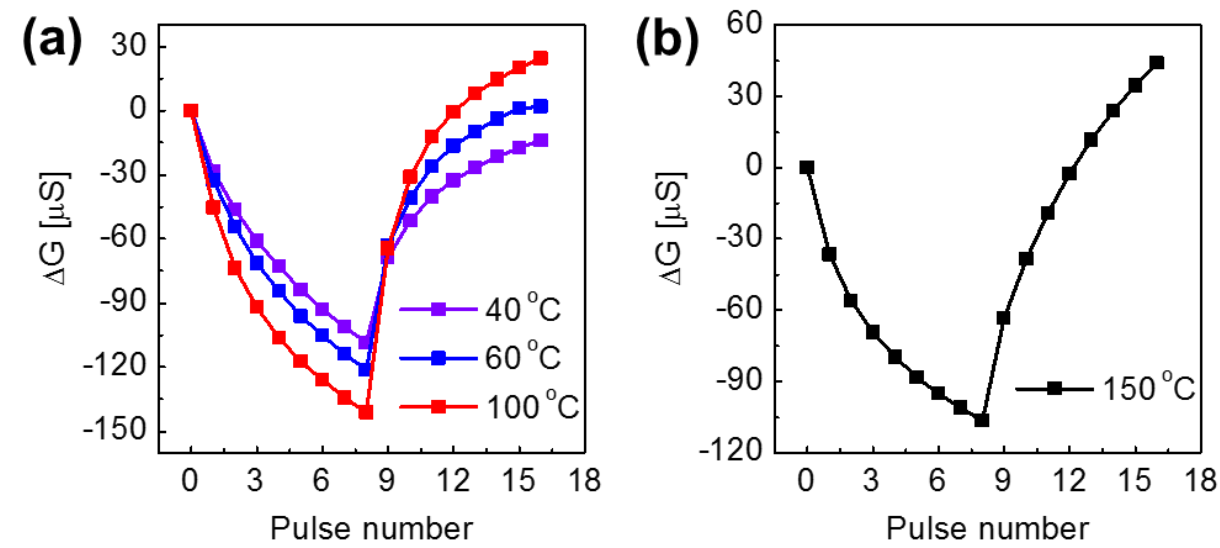

Figure S6 | Synaptic behavior at different temperature up to $150{ }^{\circ} \mathrm{C}$. 